[8] J. N. Laneman, D. N. C. Tse, and G. W. Wornell, "Cooperative diversity in wireless networks: Efficient protocols and outage behavior," IEEE Trans. Inf. Theory, vol. 50, no. 12, pp. 3062-80, Dec. 2004.

[9] J. N. Laneman and G. W. Wornell, "Distributed space-time coded protocols for exploiting cooperative diversity in wireless networks," IEEE Trans. Inf. Theory, vol. 49, no. 10, pp. 2415-2525, Oct. 2003.

[10] R. U. Nabar, H. Bölcskei, and F. W. Kneubühler, "Fading relay channels: Performance limits and space-time signal design," IEEE J. Sel. Areas Commun., vol. 22, no. 6, pp. 1099-1109, Aug. 2004.

[11] L. Ozarow, S. Shamai (Shitz), and A. Wyner, "Information theoretic considerations for cellular mobil radio," IEEE Trans. Veh. Technol., vol. 43, no. 2, pp. 359-738, May 1994.

[12] N. Prasad and M. K. Varanasi, "Diversity and multiplexing tradeoff bounds for cooperative diversity protocols," in Proc. Int. Symp. Information Theory, Chicago, IL, Jun./Jul. 2004, p. 268.

[13] A. Sendonaris, E. Erkip, and B. Aazhang, "User cooperation diversityPart I: System description,” IEEE Trans. Commun., vol. 51, no. 11, pp. 1927-38, Nov. 2003.

[14] I. E. Teletar, "Capacity of multi-antenna Gaussian channels," Europ. Trans. Telecommun., vol. 10, pp. 585-596, Nov./Dec. 1999.

[15] D. N. C. Tse, P. Viswanath, and L. Zheng, "Diversity-multiplexing tradeoff in multiple access channels," IEEE Trans. Inf. Theory, vol. 50, no. 9, pp. 1859-1874, Sep. 2004.

[16] L. Zheng and D. N. C. Tse, "Diversity and multiplexing: A fundamental tradeoff in multiple antenna channels," IEEE Trans. Inf. Theory, vol. 49, no. 5, pp. 1073-1096, May 2003.

\section{On Outer Bounds to the Capacity Region of Wireless Networks}

Sahand Haji Ali Ahmad, Aleksandar Jovičić, Student Member, IEEE, and Pramod Viswanath, Member, IEEE

\begin{abstract}
In this correspondence, we study the capacity region of a general wireless network by deriving fundamental upper bounds on a class of linear functionals of the rate tuples at which joint reliable communication can take place. The widely studied transport capacity is a specific linear functional: the coefficient of the rate between a pair of nodes is equal to the Euclidean distance between them. The upper bound on the linear functionals of the capacity region is used to derive upper bounds to scaling laws for generalized transport capacity: the coefficient of the rate between a pair of nodes is equal to some arbitrary function of the Euclidean distance between them, for a class of minimum distance networks. This upper bound to the scaling law meets that achievable by multihop communication over these networks for a wide class of channel conditions; this shows the optimality, in the scaling-law sense, of multihop communication when studying generalized transport capacity of wireless networks.
\end{abstract}

Index Terms-Ad hoc wireless networks, capacity region, cut-set bounds, isometric embedding, multihop, transport capacity.

\section{INTRODUCTION}

A characterization of the capacity region of wireless networks is one of the long standing open problems in information theory. Progress in

Manuscript received February 12, 2005; revised April 25, 2005. This work was supported in part by the National Science Foundation by Grant CCR-0312413 and by a Grant from Motorola, Inc., as part of the Motorola Center for Communication.

The authors are with the Department of Electrical and Computer Engineering, University of Illinois at Urbana-Champaign, Urbana, IL 61801 USA (e-mail: alihaji@uiuc.edu; jovicicuiuc.edu; pramodv@uiuc.edu).

Communicated by R. W. Yeung, Guest Editor.

Digital Object Identifier 10.1109/TIT.2006.874533 this direction has recently come by relaxing the questions asked: focus has been on the transport capacity [3], a specific linear functional of rate tuples at which jointly reliable communication is possible. The coefficient of the rate between a pair of nodes in this linear functional is equal to the Euclidean distance between the two nodes. Further, much emphasis is on the scaling law of the transport capacity as a function of the number of nodes in the network. Recent results have shown that multihop communication is scaling-law optimal for a class of minimum distance networks provided the wireless channel decays fast enough [4], [7], [8]. A study of the sum capacity of the wireless network appears in [5].

In an effort to understand the entire capacity region of the wireless network, we shift the focus from transport or sum capacity to studying arbitrary linear functionals of the rate tuples in the capacity region. The capacity region is a compact convex set. Thus, understanding the extremum of linear functionals of the rate tuples in the capacity region is an alternative but equivalent characterization (the convex dual in the language of convex analysis; see [6]) of the capacity region. In this correspondence, we derive simple and robust upper bounds to a class of linear functionals: the upper bound simply depends on the distance between the nodes, channel attenuation conditions and the coefficients of the linear functional. The key technique is a combination of the results of isometric embeddability of an arbitrary metric space into the $l_{1}$ metric space and the familiar information-theoretic cut-set upper bounds to the capacity region.

We use this simple upper bound to exactly characterize the scaling law behavior over arbitrary linear and planar networks of generalized transport capacity

$$
C_{f} \stackrel{\text { def }}{=} \max _{\left(R_{i j}\right) \text { in the capacity region }} \sum_{i, j=1}^{n} R_{i j} f\left(r_{i j}\right)
$$

where and $R_{i j}$ and $r_{i j}$ denote, respectively, the rate of reliable communication and the Euclidean distance between a pair of nodes $(i, j)$. When $f(\cdot)$ is the identity function, i.e., $f(x)=x$, we get back the transport capacity. The scaling law of generalized transport capacity depends crucially on the long range behavior of the function $f(\cdot)$; of particular interest is the parameter

$$
\alpha_{f} \stackrel{\text { def }}{=} \varlimsup_{x \rightarrow \infty}\left(\frac{\log f(x)}{\log x}\right) .
$$

To be able to state the scaling law precisely, we briefly describe our wireless channel model adopted from [4]: the received signal at node $j$ at time $m$ is

$$
y_{j}[m]=\sum_{i \neq j} \frac{h_{i j}^{s}[m]}{\left(1+r_{i j}\right)^{\delta}} x_{i}[m]+z_{j}[m] .
$$

Here $x_{i}[m]$ is the signal transmitted by node $i$ at time $m$ and $z_{j}[m]$ is i.i.d. white Gaussian noise. Each node $j$ has an average transmit power constraint (denoted by $P_{j}$ ). The fading channel $\left\{h_{i j}^{s}[m]\right\}_{m}$ is a stationary and ergodic zero-mean stochastic process that is independent for each pair of nodes $(i, j)$ and models the small scale fluctuations of frequency flat fading. For simplicity, we assume that $\mathbb{E}\left[\left|h_{i j}^{s}[m]\right|^{2}\right]=$ 1 for all $i, j, m$. The large scale variations are modeled explicitly ${ }^{1}$ through the decay of signal level: a factor of $\frac{1}{\left(1+r_{i j}\right)^{\delta}}$ from node $i$ to node $j$. In free space $\delta=1$, with a single reflected path along with the

${ }^{1}$ The far field signal decay is usually denoted by $r_{i j}^{-\delta}$. Here, we have written $\left(1+r_{i j}\right)^{-\delta}$ to ensure that our model makes sense when nodes get close; i.e., the average received power is not more than the average transmit power. 
line of sight it becomes equal to two and it can be larger than 2.5 in crowded urban environments.

As in [4], we consider two (disparate) models of the wireless fading channel. If the channel variations are "slow" enough so that coherent communication is possible, we model this scenario by allowing full knowledge at each node of the fading channels from all the other nodes. This is also known as the full-CSI scenario (channel state information at both the transmitter and receiver nodes). In the scenario where the channel is changing rapidly enough so that coherent communication is not feasible, our model is to assume complete ignorance of the channel realizations by all the nodes. This is the no CSI model. Clearly these are two extreme models but they serve to cover the ranges of channel fluctuations and allow us to make simple statements about the network communication problem.

Our main results are as follows.

1) The generalized transport capacity of minimum distance networks with $n$ nodes on a line is upper bounded by $K_{1} n$ for $\delta>1+0.25 \alpha_{f}$, as long as $\alpha_{f} \leq 1$. With no CSI, the same bound holds when $\delta>0.5+0.5 \alpha_{f}$ and $\alpha_{f} \leq 1$.

2) The generalized transport capacity of minimum distance networks with $n$ nodes on a plane is upper bounded by $K_{2} n$ for $\delta>2+0.25 \alpha_{f}$, as long as $\alpha_{f} \leq 1$. With no CSI, the same bound holds with $\delta>1+0.5 \alpha_{f}$ and $\alpha_{f} \leq 1$.

With multihop communication, the generalized transport capacity is lower bounded by $K_{0} n$ [8], so the upper bounds to the scaling law we derive are actually tight. This shows the optimality, in the sense of the scaling-law of the generalized transport capacity, of operating the wireless network in the multihop communication mode. These results also strictly strengthen the scaling law characterization of the transport capacity in [4] and [7], when $f(r)=r$ : the bounds therein require at least $\delta>1.5$ for linear networks and $\delta>2.5$ for planar networks with full CSI for linear scaling to hold. Further, our result is robust to power fluctuations in the network: in particular, the result continues to hold even when only the total power in the network is allowed to grow linearly with the number of nodes.

Finally, when the function $f(\cdot)$ is superlinear, if $\alpha_{f}>\delta$ then the generalized transport capacity of minimum distance linear networks grows superlinearly with the number of nodes.

We have organized the results into two parts. First, we derive an upper bound to a class of linear functionals (where the coefficients of the linear functional constitute a metric space) of rate tuples in the capacity region of an arbitrary wireless network; this is done in Section II. Second, we use this upper bound to derive the optimal scaling law of the generalized transport capacity of minimum distance wireless networks; this is done in Section III.

\section{UPPER BOUNDS TO LINEAR FUNCTIONALS OVER THE CAPACITY REGION}

We are interested in the extremum of linear functionals over the capacity region

$$
C_{\left\{a_{i j}\right\}} \stackrel{\text { def }}{=} \max _{\left(R_{i j}\right) \in \text { capacity region }} \sum_{i, j=1}^{n} a_{i j} R_{i j}, \quad a_{i j} \geq 0
$$

where there are $n$ nodes in the network $\mathcal{N} \stackrel{\text { def }}{=}\{1, \ldots, n\}$. Our interest stems from the fact that the collection of the extremum values $C_{\left\{a_{i j}\right\}}$ for all $a_{i j} \geq 0$ is a precise representation (the convex dual) of the capacity region. Here, we derive upper bounds to $C_{\left\{a_{i j}\right\}}$ for a class of linear functionals represented by $\left\{a_{i j}\right\}$. There are two key ideas that we use.

1) Given any disjoint partition (or cut) of the network (i.e., $\mathcal{C}^{+}$, $\mathcal{C}^{-} \subseteq \mathcal{N}$, such that $\mathcal{C}^{+} \cap \mathcal{C}^{-}=\emptyset$ and $\mathcal{N}=\mathcal{C}^{+} \cup \mathcal{C}^{-}$), we can bound the sum-rate at which the nodes in $\mathcal{C}^{+}$can reliably communicate to the nodes in $\mathcal{C}^{-}$by the well-known cut-set bound ([1, Theorem 14.10.1]).

2) We weight the different cuts appropriately: we want the probability that a pair of nodes $(i, j)$ gets cut to be proportional to $a_{i j}$. Then, we can average the bound on the sum-rate over all the possible cuts to arrive at an upper bound to the linear functional of interest.

The first step can be achieved for any cut of the network. However, the second step can be achieved only for a certain specific set of coefficients $\left\{a_{i j}\right\}$. In particular, the set of coefficients needs to satisfy a geometric property: there has to be some dimension $m$ such that $n$ points can be positioned in $\mathbb{R}^{m}$ with the property that the set of $l_{1}$ distances $^{2}$ between every pair of points exactly coincides with the set of coefficients $\left\{a_{i j}\right\}$. Mathematically, this requirement is stated as the following. The set of $\left\{a_{i j}\right\}$ is isometrically embeddable in $l_{1}$. This is an important result from the theory of metric spaces and we state this as a lemma.

Lemma 2.1 ([2, Proposition 4.2.2 ]): The set of distances $\left\{a_{i j}, i, j \in \mathcal{N}\right\}$ is isometrically embeddable in $l_{1}$ if and only if there exist nonnegative numbers

$$
\left\{P_{\left\{\mathcal{C}^{+}, \mathcal{C}^{-}\right\}}, \mathcal{C}^{+} \subseteq \mathcal{N} \text { and } \mathcal{C}^{-}=\mathcal{N}-\mathcal{C}^{+}\right\}
$$

such that

$$
a_{i j}=\sum_{\mathcal{C}+\subseteq \mathcal{N}: i \in \mathcal{C}+, j \in \mathcal{C}-} P_{\{\mathcal{C}+, \mathcal{C}-\}}
$$

One implication of this result is that any nonnegative linear combination of isometrically $l_{1}$-embeddable coefficent sets $\left\{a_{i j}\right\}$ is also isometrically $l_{1}$-embeddable. Apart from this general observation, the embeddability condition imposes some obvious conditions on the coefficients $\left\{a_{i j}\right\}$; in particular, they must be the distances of some $n$-point metric space. In other words

1) $a_{i i}=0$; this is, however, not a serious limitation since we do not intend to allow communication from a node to itself and there is no loss in generality in supposing the coefficient of $R_{i i}$ to be zero.

2) $a_{i j}=a_{j i}$; so we are weighting the rate from node $i$ to $j$ equally with the rate from node $j$ to $i$.

3) $a_{i j}+a_{i k} \geq a_{j k}$; the triangle inequality.

For $n \leq 4$ nodes, the above three conditions are sufficient as well for the set $\left\{a_{i j}\right\}$ to be isometrically embeddable in $l_{1}$. For large $n$, the problem of determining when a set of distances is isometrically embeddable in $l_{1}$ is computationally hard: it is NP-complete ([2, p. 49]). For values of $n \leq 7$, closed form necessary and sufficient conditions are available in [2, pp. 503-506]. Our interest, however, is to use this result only as an intermediary step in deriving an upper bound to the linear functional $C_{\left\{a_{i j}\right\}}$; thus the computational issues are hardly of any concern here.

Now we are ready to state and prove the main theorem of this section: an upper bound to $C_{\left\{a_{i j}\right\}}$. Suppose each node $i$ in the network $\mathcal{N}=$ $\{1, \ldots, n\}$ is power limited to $P_{i}$.

Theorem 2.2: Consider an arbitrary configuration $\mathcal{N}$ of $n$ nodes in the plane. Assume that the set $\left\{a_{i j}\right\}$ is isometrically $l_{1}$-embeddable. With no channel state information (CSI) at the nodes

$$
C_{\left\{a_{i j}\right\}}<\sum_{i, j=1}^{n} P_{i} \frac{a_{i j}}{\left(1+r_{i j}\right)^{2 \delta}} .
$$

${ }^{2}$ The $l_{1}$ distance between a pair of points in $\mathbb{R}^{m}$ is the sum of the absolute value of the distances between the pair of points in each of the $m$ dimensions. 
With full CSI at the nodes

$$
\begin{array}{r}
C_{\left\{a_{i j}\right\}}<\sum_{\substack{i, k=1 \\
i \neq k}}^{n} \sqrt{P_{i} P_{k}}\left\{\sum_{j=1}^{n} \frac{\min \left(a_{i j}, a_{k j}\right)^{2}}{\left(1+r_{i j}\right)^{2 \delta}\left(1+r_{k j}\right)^{2 \delta}}\right\}^{\frac{1}{2}}, \\
+\sum_{i, j=1}^{n} P_{i} \frac{a_{i j}}{\left(1+r_{i j}\right)^{2 \delta}}
\end{array}
$$

Remark: Since the second term in (6) is exactly the no-CSI bound in (5), the significance of the first term in (6) is that it is a bound on the communication gain of having CSI at all the nodes.

The proof of Theorem 2.2 is relegated to Appendix I.

\section{A. Examples of Linear Functionals}

1) Rate Coefficients as Functions of Distance: If we substitute $a_{i j}=r_{i j}$, the Euclidean distance $\left(l^{2}\right.$ distance) between the pair of nodes $(i, j)$, we see that (6) is a strengthening of (6) in $[4$, Theorem 2.1]: the improvement in the bound comes from using a single fixed distribution of network inputs for all possible cuts of the network (see Appendix I). The bound given in (5) remains the same as (5) in [4, Theorem 2.1].

For this special choice of coefficients, $a_{i j}=r_{i j}$, we can then use a random line, as in [4], to geographically partition the network so that the probability that any pair of nodes is cut by the line is proportional to the Euclidean distance between the nodes. Since a set of distances that are embeddable in the $l^{2}$ metric space are isometrically embeddable in the $l_{1}$ metric space ([2, Proposition 6.4.12]), we can cast the utility of the random line construction in [4] as a special case of Lemma 2.1. In particular, the probability that a pair of nodes $(i, j)$ is cut using the random line is (cf. [4, eq. 10])

$$
\mathbb{P}\left[i \in \mathcal{C}^{+}, j \in \mathcal{C}^{-}\right]=\frac{r_{i j}}{2 \pi d_{n}}
$$

where $d_{n}$ is the radius of a circle that contains all the $n$ points in the network. To arrive at the statement of Theorem 2.2 we choose the probabilities of the cuts explicitly so that (7) is replaced by the implication from Lemma 2.1

$$
\mathbb{P}\left[i \in \mathcal{C}^{+}, j \in \mathcal{C}^{-}\right]=\frac{a_{i j}}{A}
$$

for some constant $A$ chosen such that the quantity on the left side of the equation above, summed over all possible cuts, sums to unity. The proof of (6) in Theorem 2.2 uses these observations and is given in Appendix I.

An important class of coefficients $\left\{a_{i j}\right\}$ that is isometrically $l_{1}$-embeddable is the following ([2, p. 114]):

$$
a_{i j}=r_{i j}^{\alpha}, \quad 0 \leq \alpha \leq 1
$$

In particular, $\alpha=0$ corresponds to $a_{i j}=1$ for each pair of nodes $(i, j)$ and thus the corresponding linear functional is simply the sum capacity of the network.

Further, for any density function $g(\cdot)$

$$
a_{i j}=\int_{0}^{1} r_{i j}^{\alpha} g(\alpha) d \alpha
$$

is also isometrically embeddable; this is a special case of the earlier observation that nonnegative linear combinations of isometrically $l_{1}$ embeddable coefficients also are isometrically $l_{1}$ embeddable. We use this result to study the scaling law behavior of generalized transport capacity in Section III.

2) Extension to an Arbitrary Metric Space: Up until now, we have required that the finite metric space whose distances are given by $\left\{a_{i j}\right\}$ be isometrically embeddable into an $l_{1}$ space, i.e., that the distances be exactly preserved. However, if we allow embeddings that introduce some relative distortion in the distances, we can extend our upper bounds to any linear functional as long as its coefficients are distances in some metric space.

Lemma 2.3 (Bourgain, 1985, See [2, Theorem 10.1.2]): Suppose $\left\{a_{i j}\right\}$ is the distance between $x_{i}$ and $x_{j}$ in some $n$ point metric space $\mathcal{N}$. There exists an embedding $f: \mathcal{N} \rightarrow \mathbb{R}^{m}$ such that

$$
\left\|f\left(x_{i}\right)-f\left(x_{j}\right)\right\|_{1} \leq a_{i j} \leq c_{0} \log n\left\|f\left(x_{i}\right)-f\left(x_{j}\right)\right\|_{1}
$$

for all $x, y \in \mathcal{N}$, where \|\|$_{1}$ denotes the $l_{1}$ norm and $c_{0}<92$ is a constant.

This theorem allows us to state the following corollary to Theorem 2.2.

Corollary 2.1: Consider an arbitrary configuration $\mathcal{N}$ of $n>1$ nodes in the plane. Assume that the set $\left\{a_{i j}\right\}$ represents distances in some metric space. With no channel state information (CSI) at the nodes

$$
\frac{C_{\left\{a_{i j}\right\}}}{c_{0} \log n}<\sum_{i, j=1}^{n} P_{i} \frac{a_{i j}}{\left(1+r_{i j}\right)^{2 \delta}}
$$

With full CSI at the nodes

$$
\begin{aligned}
\frac{C_{\left\{a_{i j}\right\}}}{c_{0} \log n}<\sum_{i, k=1}^{n} \sqrt{P_{i} P_{k}}\left\{\sum_{j=1}^{n} \frac{\min \left(a_{i j}, a_{k j}\right)^{2}}{\left(1+r_{i j}\right)^{2 \delta}\left(1+r_{k j}\right)^{2 \delta}}\right\}^{\frac{1}{2}} & \\
& +\sum_{i, j=1}^{n} P_{i} \frac{a_{i j}}{\left(1+r_{i j}\right)^{2 \delta}} .
\end{aligned}
$$

The proof of this corollary follows the proof of Theorem 2.2 (Appendix I) along with the following observation: by Lemma 2.3, $\sum_{i j} R_{i j} a_{i j} \leq c_{0} \log n \sum_{i j} R_{i j} \tilde{a}_{i j}$, where $\tilde{a}_{i j}:=\left\|f\left(x_{i}\right)-f\left(x_{j}\right)\right\|_{1}$. Now, Lemma 2.1 guarantees that there exists a distribution on the cut-sets $\left(\mathcal{C}^{+}, \mathcal{C}^{-}\right)$for which $\mathbb{P}\left[i \in \mathcal{C}^{+}, j \in \mathcal{C}^{-}\right]$is proportional to $\tilde{a}_{i j}$. Averaging over this distribution and using the left-hand inequality in Lemma 2.3, we obtain the desired result.

3) Metric Spaces Defined on Planar Graphs: Lemma 2.1 states that it is possible to embed any metric space into $l_{1}$ with distortion at $\operatorname{most} O(\log n)$. However, if the metric space in question is defined on a certain type of planar graph, there exist $l_{1}$ embeddings with distortion that is independent of $n$.

In particular, a graph metric ${ }^{3}$ defined on any treewidth- 2 planar graph can be embedded into $l_{1}$ with distortion of at most 14 (see [9, Theorem 4.1]). Hence, for such metric spaces, we can strengthen the statement of Corollary 2.1 by replacing the $\log n$ factor with the constant 14 . The long-standing conjecture is that every metric defined on a planar graph can be embedded into $l_{1}$ with constant distortion.

Moreover, the set of all planar graphs whose metrics are isometrically $l_{1}$ embeddable (i.e., embeddable with unit distortion) is exactly the set of planar graphs that do not contain $K_{2,3}$ (Fig. 1) as a minor (see [9, Proposition 3.1]). For such metric spaces, we can directly apply Theorem 2.2.

${ }^{3}$ The distance between any two nodes is the smallest sum of (nonnegative) weights on any path between them. 


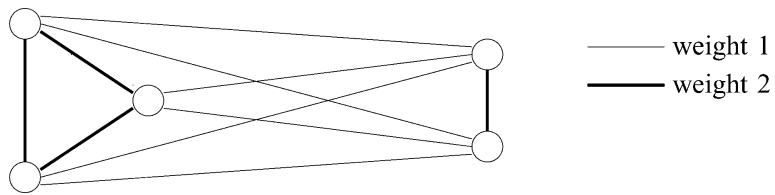

Fig. 1. It can be shown (see [2, Example 6.3.2]) that the metric space generated by this complete, bipartite graph (also known as $K_{2,3}$ ) is not isometrically embeddable in $l_{1}$.

\section{SCALING LAWS FOR GENERALIZED TRANSPORT CAPACITY}

Our upper bound to the extremum of linear functionals of the capacity region was for arbitrary network topologies. Here we focus on a specific network topology-minimum distance networks-to study the scaling law behavior of generalized transport capacity. We consider both linear and planar networks with minimum distance between the nodes denoted by $r_{m}>0$. Suppose the average power constraint of each radio is uniformly upper bounded by $P$. Our main result is the following: the generalized transport capacity scales linearly with the number of nodes $n$, provided:

1) the function $f(r)$ grows sublinearly with the Euclidean distance $r$, i.e., $\alpha_{f} \leq 1$ (cf. (2)), and

2) the channel decays fast enough, relative to the growth of the function $f(r)$.

Theorem 3.1: Suppose the nodes lie on a line at a distance of at least $r_{m}>0$ from each other.

1) With no CSI at the nodes the generalized transport capacity is upper bounded by

$$
C_{f}<\frac{2 P \zeta\left(2 \delta-\alpha_{f}\right)}{r_{m}^{2 \delta-\alpha_{f}}} n
$$

for all $\delta>\frac{1+\alpha_{f}}{2}$ and $\alpha_{f} \leq 1$.

2) With full CSI at the nodes, the generalized transport capacity is upper bounded by

$$
C_{f}<\frac{P}{r_{m}^{2 \delta-\alpha_{f}}}\left\{G\left(\alpha_{f}, \delta\right)+2 \zeta\left(2 \delta-\alpha_{f}\right)\right\} n
$$

as long as $\delta>1+\frac{\alpha_{f}}{4}$ and $\alpha_{f} \leq 1$.

Here, we have defined the constant

$$
\begin{aligned}
G\left(\alpha_{f}, \delta\right) \stackrel{\text { def }}{=} 4\left\{\zeta\left(2 \delta-\alpha_{f}\right)+K_{1}\left(\alpha_{f}, \delta\right)^{\frac{1}{2}} \zeta\left(2 \delta-\alpha_{f}-\frac{1}{2}\right)\right. \\
+2^{\delta-\frac{\alpha_{f}}{4}}\left[\zeta\left(\delta-\frac{\alpha_{f}}{4}\right)+K_{1}\left(\alpha_{f}, \delta\right)^{\frac{1}{2}} c_{1}\left(\alpha_{f}, \delta\right)\right. \\
\\
\left.\left.\left(\zeta\left(\delta-\frac{\alpha_{f}}{4}\right)-\zeta\left(2 \delta-\alpha_{f}-\frac{1}{2}\right)\right)\right]\right\}
\end{aligned}
$$

where $\zeta(\delta) \stackrel{\text { def }}{=} \sum_{i=1}^{\infty} i^{-\delta}$ is the Riemann-Zeta function (a finite number for $\delta>1$ )

$$
c_{1}\left(\alpha_{f}, \delta\right) \stackrel{\text { def }}{=}\left(1-2^{\frac{3}{2} \alpha_{f}-2 \delta+1}\right)^{\frac{1}{2}}\left(1-2^{\frac{3}{4} \alpha_{f}-\delta+\frac{1}{2}}\right)^{-1}
$$

and

$$
K_{1}\left(\alpha_{f}, \delta\right) \stackrel{\text { def }}{=}\left(2 \delta-\frac{3}{2} \alpha_{f}-1\right)^{-1}
$$

Theorem 3.2: For planar networks with a distance of at least $r_{m}>0$ between any two nodes, the scaling law is similar

1) with no CSI at the nodes, the generalized transport capacity is upper bounded by

$$
C_{f}<\frac{(2 \pi+12) P \zeta\left(2 \delta-\alpha_{f}-1\right)}{r_{m}^{2 \delta-\alpha_{f}}} n
$$

for $\delta>1+\frac{\alpha_{f}}{2}$ and $\alpha_{f} \leq 1$;

2) with full CSI at the nodes, the generalized transport capacity is upper bounded by

$$
C_{f}<\frac{P n}{r_{m}^{2 \delta-\alpha_{f}}}\left\{H\left(\alpha_{f}, \delta\right)+(2 \pi+12) \zeta\left(2 \delta-\alpha_{f}-1\right)\right\}
$$

for $\delta>2+\frac{\alpha_{f}}{4}$ and $\alpha_{f} \leq 1$.

Here, we have defined the constant as shown in the equation at the bottom of the page, where $\zeta(\delta)$ is as defined in Theorem 3.1

$$
K_{2}\left(\alpha_{f}, \delta\right) \stackrel{\text { def }}{=}\left(2 \delta-\frac{3}{2} \alpha_{f}-2\right)^{-1}
$$

and

$$
c_{2}\left(\alpha_{f}, \delta\right) \stackrel{\text { def }}{=}\left(1-2^{\frac{3}{2} \alpha_{f}-2 \delta+2}\right)^{\frac{1}{2}}\left(1-2^{\frac{3}{4} \alpha_{f}-\delta+1}\right)^{-1} .
$$

The first step in the proofs of the above two theorems, an upper bound to the linear functional of interest, has been generalized for a class of coefficients $\left\{a_{i j}\right\}$ in Theorem 2.2. Since the choice of coefficients $a_{i j}=r_{i j}^{\alpha}$ for $\alpha \leq 1$ is isometrically $l_{1}$-embeddable [see (9)], the bound in Theorem 2.2 can be used to evaluate an upper bound to the generalized transport capacity for functions of the form $f(r)=r^{\alpha}$ for $\alpha \leq 1$. The proof of the full-CSI cases is given in Appendices II and III. The no-CSI case is treated very similarly to Corollaries 3.1 and 3.2 in [4], except that the conditions on the signal decay parameter $\delta$ now depend $\alpha_{f}$.

Remark 1: For the special case of $a_{i j}=r_{i j}$, the result of Theorem 2.2 is a strict improvement over the previous results on the scaling behavior of transport capacity, [4], [7]: the bounds therein require at least $\delta>1.5$ for linear networks and $\delta>2.5$ for planar networks with full CSI for linear scaling to hold.

$$
\begin{aligned}
H\left(\alpha_{f}, \delta\right) \stackrel{\text { def }}{=} & \sqrt{2}(2 \pi+12)^{\frac{3}{2}}\left\{\zeta\left(2 \delta-\alpha_{f}-\frac{3}{2}\right)+K_{2}\left(\alpha_{f}, \delta\right)^{\frac{1}{2}} \zeta\left(2 \delta-\alpha_{f}-2\right)+2^{\delta-\frac{\alpha_{f}}{4}}\right. \\
& \left.\times\left[\zeta\left(\delta-\frac{\alpha_{f}}{4}-1\right)+K_{2}\left(\alpha_{f}, \delta\right)^{\frac{1}{2}} c_{2}\left(\alpha_{f}, \delta\right)\left(\zeta\left(\delta-\frac{\alpha_{f}}{4}-1\right)-\zeta\left(2 \delta-\alpha_{f}-2\right)\right)\right]\right\}
\end{aligned}
$$


Remark 2: When $a_{i j}=1$ for all $i, j$, we establish the optimality of multihop with respect to sum-capacity of the network, for $\delta>1$ in a linear and $\delta>2$ in a planar network. Sum capacity bounds for a particular traffic pattern (across a single cut of the network) have been studied in [5]. Our results hold regardless of the assumptions on the traffic pattern.

Remark 3: Examining the origin of the constant factors in the bounds of Theorems 3.1 and 3.2, we observe that it is the communication between "nearby" nodes that determines the requirement on the path-loss parameter for linear scaling to hold. This vicinity is quantified in the proofs.

So far, we have only considered functions $f(\cdot)$ that are sub-linear in $n$. What happens when the function is superlinear in $n$ ? It turns out that in this case, the generalized transport capacity can grow faster than the number of nodes in the network. The communication strategy that achieves this is coherent multistage relaying and interference cancellation described in [7, Theorem 4.3]. The following statement, based directly on [7, Theorem 3.6], makes this precise:

Proposition 3.1: The generalized transport capacity for linear minimum-distance networks can grow super-linearly with the number of nodes $n$, according to $\Omega\left(n^{\alpha_{f} / \delta}\right)$ as long as $\alpha_{f}>\delta$. This scaling law is also optimal when $\frac{1}{2}<\delta<1$ and $\alpha_{f}>\delta$.

\section{APPENDIX I}

\section{PROOF OF THE FULL-CSI CASE IN THEOREM 2.2}

The cut-set bound ([1, Theorem 14.10.1 ]) applied to a network $\mathcal{N}$ with the channel model given in (3) and with full CSI at all the nodes can be stated in the following way: There exists a $n \times n$ covariance matrix $\mathbf{Q}$ (with $(\mathbf{Q})_{i i} \leq P_{i}$ for all $i \in \mathcal{N}$ ) such that the sum-rate at which the nodes in $\mathcal{C}^{+}$can reliably communicate to the nodes in $\mathcal{C}^{-}$ satisfies

$$
\sum_{i \in \mathcal{C}^{+}, j \in \mathcal{C}^{-}} R_{i j}<\log \operatorname{det}\left(\mathbf{I}+\mathbf{H}_{\mathcal{C}} \mathbf{Q}_{\mathcal{C}} \mathbf{H}_{\mathcal{C}}^{\dagger}\right)
$$

for every disjoint partition $\mathcal{C}=\left(\mathcal{C}^{+}, \mathcal{C}^{-}\right)$of $\mathcal{N}$, where the $\left|\mathcal{C}^{+}\right| \times$ $\left|\mathcal{C}^{+}\right|$matrix $Q_{\mathcal{C}}$ is obtained by retaining only the elements lying in the rows and columns of $\mathbf{Q}$ that are indexed by $i \in \mathcal{C}^{+}$(i.e., it is a principal submatrix), and where the $\left|\mathcal{C}^{-}\right| \times\left|\mathcal{C}^{+}\right|$matrix $\mathbf{H}_{\mathcal{C}}$ is obtained by retaining only the rows and columns of $\mathbf{H}$ that are indexed by $j \in$
$\mathcal{C}^{-}$and $i \in \mathcal{C}^{+}$, respectively. The entries $(\mathbf{H})_{i j}$ are independent, zero mean random variables with variance $\frac{1}{\left(1+r_{i j}\right)^{2 \delta}}$. Note that the principal submatrix $\mathbf{Q}_{\mathcal{C}}$ is always positive semidefinite.

We can then further upper-bound the sum-rate by using the Hadamard bound in the log-det expression, followed by $\log (1+x) \leq x$

$$
\begin{aligned}
\sum_{i \in \mathcal{C}^{+}, j \in \mathcal{C}-} R_{i j} & <\log \operatorname{det}\left(\mathbf{I}+\mathbf{H}_{\mathcal{C}} \mathbf{Q}_{\mathcal{C}} \mathbf{H}_{\mathcal{C}}^{\dagger}\right) \\
& \leq \sum_{j \in \mathcal{C}^{-}} \log \left(1+\mathbf{h}_{j}(\mathcal{C}) \mathbf{Q}_{\mathcal{C}} \mathbf{h}_{j}^{\dagger}(\mathcal{C})\right) \\
& =\sum_{j \in \mathcal{C}^{-}} \log \left(1+\sum_{i, k \in \mathcal{C}^{+}} h_{j i}(\mathbf{Q})_{i k} h_{j k}^{*}\right) \\
& \leq \sum_{i, k=1}^{n}(\mathbf{Q})_{i k} \sum_{j=1}^{n} h_{j i} h_{j k}^{*} \mathbf{1}_{\left\{i \in \mathcal{C}+, k \in \mathcal{C}^{+}, j \in \mathcal{C}^{-}\right\}}
\end{aligned}
$$

Here we have used $\mathbf{h}_{j}(\mathcal{C})$ to denote the $j$-th row of the submatrix $\mathbf{H}_{\mathcal{C}}$, and $\mathbf{1}_{\{\}}$to denote the indicator function. Thus, we obtain an upperbound on $C_{\left\{a_{i j}\right\}}$ as shown in (10)-(14) at the bottom of the pagewhere we have used $\mathbb{E}_{\mathbf{H}}$ to denote expectation with respect to the channel statistics and $\mathbb{E}_{\mathcal{C}}$ to denote the expectation with respect to the distribution of the cuts. Observe that (11) follows from (8), and (12) follows from the fact that $(\mathbf{Q})_{i i}(\mathbf{Q})_{j j} \geq\left|(\mathbf{Q})_{i j}\right|^{2}$, since every principal minor of a positive semidefinite matrix is nonnegative. Equality (13) is obtained by summing over $i \neq k$ and $i=k$ separately. Inequality (14) is obtained by applying Jensen's inequality to the concave function $x \rightarrow x^{1 / 2}$, and making use of the independence of the $\left\{h_{i j}^{s}\right\}$ as well as their zero-mean property. This concludes the proof of (6) in Theorem 2.2.

\section{APPENDIX II}

\section{Proof OF THE FULL-CSI CASE IN THEOREM 3.1}

Let $P=\max _{i \in \mathcal{N}} P_{i}$. We focus on the case where $a_{i j}=r_{i j}^{\alpha}$, for $\alpha \leq 1$. Then we can express the first term in (6) as

$$
C_{\left\{a_{i j}\right\}}^{\mathrm{CSI} \text { gain }} \leq P \sum_{i, k=1}^{n}\left\{\sum_{j=1}^{n} \frac{\min \left(r_{j i}^{\alpha}, r_{j k}^{\alpha}\right)^{2}}{\left(1+r_{j i}\right)^{2 \delta}\left(1+r_{j k}\right)^{2 \delta}}\right\}^{\frac{1}{2}}
$$

$$
\begin{aligned}
C_{\left\{a_{i j}\right\}} & <A \mathbb{E}_{\mathbf{H}}\left[\max _{\mathbf{Q} \succeq 0:(\mathbf{Q})_{i i} \leq P_{i}} \mathbb{E}_{\mathcal{C}}\left[\sum_{i, k=1}^{n}(\mathbf{Q})_{i k} \sum_{j=1}^{n} h_{j i} h_{j k}^{*} \mathbf{1}_{\{i \in \mathcal{C}+, k \in \mathcal{C}+, j \in \mathcal{C}-\}}\right]\right] \\
& =A \mathbb{E}_{\mathbf{H}}\left[\max _{\mathbf{Q} \succeq 0:(\mathbf{Q})_{i i} \leq P_{i}} \sum_{i, k=1}^{n}(\mathbf{Q})_{i k} \sum_{j=1}^{n} h_{j i} h_{j k}^{*} \frac{\min \left(a_{j i}, a_{j k}\right)}{A}\right] \\
& \leq \mathbb{E}_{\mathbf{H}}\left[\sum_{i, k=1}^{n} \sqrt{P_{i} P_{k}}\left|\sum_{j=1}^{n} h_{j i} h_{j k}^{*} \min \left(a_{j i}, a_{j k}\right)\right|\right] \\
& =\sum_{\substack{i, k=1 \\
i \neq k}}^{n} \sqrt{P_{i} P_{k}} \mathbb{E}_{\mathbf{H}}\left[\left|\sum_{j=1}^{n} h_{j i} h_{j k}^{*} \min \left(a_{j i}, a_{j k}\right)\right|^{\frac{2}{2}}\right]+\sum_{i=1}^{n} P_{i} \mathbb{E}_{\mathbf{H}}\left[\sum_{j=1}^{n}\left|h_{j i}\right|^{2} a_{j i}\right] \\
& \leq \sum_{\substack{i, k=1 \\
i \neq k}}^{n} \sqrt{P_{i} P_{k}}\left\{\sum_{j=1}^{n} \frac{\min \left(a_{j i}, a_{j k}\right)^{2}}{\left(1+r_{j i}\right)^{2 \delta}\left(1+r_{j k}\right)^{2 \delta}}\right\}^{\frac{1}{2}}+\sum_{i, j=1}^{n} P_{i} \frac{a_{j i}}{\left(1+r_{j i}\right)^{s \delta}}
\end{aligned}
$$


Observing that $i=i^{*}:=\frac{n-1}{2}$ yields the largest inner sum (over $k$ and $j$ ) in (13), ${ }^{4}$ we can upper bound this expression by

$$
\begin{aligned}
\operatorname{Pn} & {\left[\sum_{k=1}^{n}\left\{\sum_{\substack{j: r_{j i^{*}>r_{j k}} \\
r_{j k}>r_{k i^{*}}}} \frac{2}{\left(1+r_{j i^{*}}\right)^{2 \delta}\left(1+r_{j k}\right)^{2 \delta-2 \alpha}}\right\}^{\frac{1}{2}}\right.} \\
& +\sum_{k=1}^{n}\left\{\sum_{\substack{j: r_{j i^{*}>r_{j k}} \\
r_{j k}<r_{k i^{*}}}} \frac{2}{\left(1+r_{j i^{*}}\right)^{2 \delta}\left(1+r_{j k}\right)^{2 \delta-2 \alpha}}\right\} .
\end{aligned}
$$

Since the summations over $j$ are symmetric around the midpoint between node $k$ and node $i^{*}$, there is a factor of 2 multiplying both of the inner sums (over $j$ ) above. In additon to splitting the above summations, we have also used $(a+b)^{\frac{1}{2}} \leq a^{\frac{1}{2}}+b^{\frac{1}{2}}$. Henceforth, we will use $B_{1}$ and $B_{2}$ to denote the first and second terms, respectively, in the square brackets in (15).

We first focus on the term $B_{1}$ above, and observe that $r_{j i}>r_{j k}$ and $r_{j k}>r_{k i *}$ imply that $r_{j i *}>r_{k i *}$, so

$$
\left(1+r_{j i^{*}}\right)^{2 \delta} \geq\left(1+r_{j k}\right)^{\frac{\alpha}{2}}\left(1+r_{k i^{*}}\right)^{2 \delta-\frac{\alpha}{2}},
$$

as long as $\delta>\frac{\alpha}{4}$. Substituting this calculation and using the fact that $\delta, \alpha \geq 0$ we upper bound the term $B_{1}$ by

$$
\begin{aligned}
& \sum_{k=1}^{n}\left\{\sum_{\substack{j: r_{j i^{*}>r_{j k}} \\
r_{j k}>r_{k i^{*}}}} \frac{2}{\left(1+r_{k i^{*}}\right)^{2 \delta-\frac{\alpha}{2}}\left(1+r_{j k}\right)^{2 \delta-2 \alpha+\frac{\alpha}{2}}}\right\}^{\frac{1}{2}} \\
& <\sum_{k=1}^{n} \frac{\sqrt{2}}{\left(1+r_{k i^{*}}\right)^{\delta-\frac{\alpha}{4}}}\left\{\sum_{j: r_{j k}>r_{k i^{*}}} \frac{1}{\left(1+r_{j k}\right)^{2 \delta-2 \alpha+\frac{\alpha}{2}}}\right\}^{\frac{1}{2}} .
\end{aligned}
$$

Now, let $l_{k i *}:=\left\lfloor r_{\left.k i^{*} / r_{m}\right\rfloor}\right.$ and $l_{j k}:=\left\lfloor r_{j k} / r_{m}\right\rfloor$. Clearly, for a given $i^{*}, l_{k i^{*}} \geq 1$ for all $k \in \mathcal{N}$ and for a given $k, l_{j k} \geq 1$ for all $j \in \mathcal{N}$. The largest number of nodes $k$ which have a common value $l_{k i}$ is simply 2 since no two nodes can be closer than $r_{m}$ and they all lie on a line. Thus, $B_{1}$ is upper bounded by

$$
\sum_{l_{k i *}=1}^{\infty} \frac{2 \sqrt{2}}{\left(1+r_{m} l_{k i^{*}}\right)^{\delta-\frac{\alpha}{4}}}\left\{\sum_{l_{j k}=l_{k i *}}^{\infty} \frac{2}{\left(1+r_{m} l_{j k}\right)^{2 \delta-\frac{3}{2} \alpha}}\right\}^{\frac{1}{2}} .
$$

Note that the inner summation (over $l_{j k}$ ) in the expression is simply a lower Riemann sum. Thus, we can further upper bound it as follows:

$$
\begin{aligned}
& \left\{\sum_{l_{j k}=l_{k i^{*}}}^{\infty} \frac{2}{\left(1+r_{m} l_{j k}\right)^{2 \delta-\frac{3}{2} \alpha}}\right\}^{\frac{1}{2}} \\
& \quad<\frac{\sqrt{2}}{r_{m}^{\delta-\frac{3}{4} \alpha}}\left\{\frac{1}{l_{k i^{*}}^{2 \delta-\frac{3}{2} \alpha}}+\int_{l_{k i^{*}}}^{\infty} \frac{d x}{x^{2 \delta-\frac{3}{2} \alpha}}\right\}^{\frac{1}{2}} \\
& =\frac{\sqrt{2}}{r_{m}^{\delta-\frac{3}{4} \alpha}}\left\{\frac{1}{l_{k i^{*}}^{2 \delta-\frac{3}{2} \alpha}}+\frac{K_{1}(\alpha, \delta)}{l_{k i^{*}}^{2 \delta-\frac{3}{2} \alpha-1}}\right\}^{\frac{1}{2}}
\end{aligned}
$$

${ }^{4}$ Since we are deriving upper bounds we can always add another node to make $n$ odd

$$
<\frac{\sqrt{2}}{r_{m}^{\delta-\frac{3}{4} \alpha}}\left\{\frac{1}{l_{k i^{*}}^{\delta-\frac{3}{\alpha} \alpha}}+\frac{K_{1}(\alpha, \delta)^{\frac{1}{2}}}{l_{k i^{*}}^{\delta-\frac{3}{2} \alpha-\frac{1}{2}}}\right\}
$$

where we have used the notation $K_{1}(\alpha, \delta):=\left(2 \delta-\frac{3}{2} \alpha-1\right)^{-1}$. Substituting this calculation in (19), we obtain the bound

$$
B_{1}<\frac{4}{r_{m}^{2 \delta-\alpha}}\left\{\zeta(2 \delta-\alpha)+K_{1}(\alpha, \delta)^{\frac{1}{2}} \zeta\left(2 \delta-\alpha-\frac{1}{2}\right)\right\}
$$

where $\zeta(\delta) \stackrel{\text { def }}{=} \sum_{i=1}^{\infty} i^{-\delta}$ is the Riemann-Zeta function and is finite for $\delta>1$. Thus, the above bound on $B_{1}$ is valid for $\delta>\frac{3}{4}+\frac{\alpha}{2}$.

Now turning to term $B_{2}$ in (15), we see that $r_{j i^{*}}>r_{j k}$ and $r_{j k}<$ $r_{k i *}$ imply that $r_{j i *}>\frac{r_{k i *}}{2}$, so (16) becomes

$$
\left(1+r_{j i^{*}}\right)^{2 \delta} \geq\left(1+r_{j k}\right)^{\frac{\alpha}{2}}\left(\frac{1+r_{k i^{*}}}{2}\right)^{2 \delta-\frac{\alpha}{2}}
$$

and the bound on $B_{2}$ is given by

$$
\begin{aligned}
& \sum_{k=1}^{n}\left(\frac{2}{1+r_{k i^{*}}}\right)^{\delta-\frac{\alpha}{4}} \\
& \quad \times\left\{\sum_{j: r_{j k}<r_{k i^{*}}} \frac{2}{\left(1+r_{j k}\right)^{2 \delta-2 \alpha+\frac{\alpha}{2}}}\right\}^{\frac{1}{2}} \\
& \left.<\sum_{l_{k i^{*}=1}^{\infty}}^{\infty} \frac{2^{1+\delta-\frac{\alpha}{4}}}{\left(1+r_{m} l_{k i^{*}}\right)^{\delta-\frac{\alpha}{4}}}\right\}^{\frac{1}{2}} . \\
& \quad \times\left\{\sum_{l_{j k}=1}^{l_{k i^{*}}} \frac{4}{\left(1+r_{m} l_{j k}\right)^{2 \delta-\frac{3}{2} \alpha}}\right\}^{\infty}
\end{aligned}
$$

Again, upper bounding the lower Riemann sum over $l_{j k}$ by its integral we get

$$
\begin{aligned}
& \left\{\sum_{l_{j k}=1}^{l_{k i^{*}}} \frac{4}{\left(1+r_{m} r_{j k}\right)^{2 \delta-\frac{3}{2} \alpha}}\right\}^{\frac{1}{2}} \\
& \leq \frac{2}{r_{m}^{\delta-\frac{3}{4} \alpha}}\left\{1+\int_{1}^{l}{ }_{k i^{*}} \frac{d x}{x^{2 \delta-\frac{3}{2} \alpha}}\right\}^{\frac{1}{2}} \\
& =\frac{2}{r_{m}^{\delta-\frac{3}{4} \alpha}}\left\{1+\frac{l_{k i^{*}}^{\frac{3}{2} \alpha-2 \delta+1}-1}{\frac{3}{2} \alpha-2 \delta+1}\right\}^{\frac{1}{2}} \\
& \leq \frac{2}{r_{m}^{\delta-\frac{3}{4} \alpha}}\left\{1+\frac{\left(1-l_{k i^{*}}^{\frac{3}{2} \alpha-2 \delta+1}\right)^{\frac{1}{2}}}{\left(2 \delta-\frac{3}{2} \alpha-1\right)^{\frac{1}{2}}}\right\}
\end{aligned}
$$

where the last line holds when $\delta>\frac{1}{2}+\frac{3}{4} \alpha$. Now we would like to find a number $c_{1}(\alpha, \delta)$, independent of $l$, such that

$$
\left(1-l^{\frac{3}{2} \alpha-2 \delta+1}\right)^{\frac{1}{2}} \leq c_{1}(\alpha, \delta)\left(1-l^{\frac{3}{4} \alpha-\delta+\frac{1}{2}}\right)
$$

for all $l=1,2, \ldots$ Observe that for $l=1$, the inequality is satisfied with $c_{1}(\alpha, \delta)=1$. Also, the function

$$
\left(1-l^{\frac{3}{2} \alpha-2 \delta+1}\right)^{\frac{1}{2}}\left(1-l^{\frac{3}{4} \alpha-\delta+\frac{1}{2}}\right)^{-1}
$$

is monotonically decreasing with $l$. Thus, we can choose

$$
c_{1}(\alpha, \delta) \stackrel{\text { def }}{=}\left(1-2^{\frac{3}{2} \alpha-2 \delta+1}\right)^{\frac{1}{2}}\left(1-2^{\frac{3}{4} \alpha-\delta+\frac{1}{2}}\right)^{-1} .
$$


Substituting this calculation in (24) we, hence, upper-bound term $B_{2}$ in (21) as follows:

$$
\begin{aligned}
B_{2}<\frac{2^{2+\delta-\frac{\alpha}{4}}}{r_{m}^{2 \delta-\alpha}}\left\{\zeta\left(\delta-\frac{\alpha}{4}\right)\right. & +K_{1}(\alpha, \delta)^{\frac{1}{2}} c_{1}(\alpha, \delta) \\
\times & {\left.\left[\zeta\left(\delta-\frac{\alpha}{4}\right)-\zeta\left(2 \delta-\alpha-\frac{1}{2}\right)\right]\right\} . }
\end{aligned}
$$

We note that the above bound on $B_{2}$ is valid only for $\delta>1+\frac{\alpha}{4}$. Observe that if $\delta>1+\frac{\alpha}{4}$, then $\delta>\frac{1}{2}+\frac{3}{4} \alpha$ thus, (24) is well defined in this regime.

Combining the two bounds on $B_{1}$ and $B_{2}$, we establish the scaling of the first term in (6). The second term exactly corresponds to the no-CSI bound of (5), whose scaling law is directly obtained from [4, Corollary 3.1], . Hence we obtain the scaling law of $C_{f}$ when $f(r)=$ $r^{\alpha}, \alpha \leq 1$ for linear minimum distance networks.

To extend this to generalized transport capacity, we make the following observation: For any general $f(\cdot)$ with $\alpha_{f}<1$ we have $f(r) \leq$ $r^{\alpha_{f}+\epsilon}$ for all $r$ sufficiently large for every $\epsilon>0$. Choosing $\epsilon$ small enough such that $\alpha_{f}+\epsilon<1$, we can upper bound the generalized transport capacity $C_{f}$ of a minimum distance network by $C_{r^{\alpha}+\epsilon}$ multiplied by a constant that does not depend on the number of nodes $n$. Since $\alpha_{f}+\epsilon \leq 1$ we can now use the scaling law result for polynomial functions of the Euclidean distance derived above to upper bound the scaling law behavior of $C_{f}$ : the scaling law behavior of $C_{f}$ is the same as that when $f(r)=r^{\alpha_{f}+\epsilon}$. Since $\epsilon>0$ can be chosen arbitrarily small, this completes the proof of Theorem 3.1.

\section{APPENDIX III}

\section{PROOF OF THE FULL-CSI CASE IN THEOREM 3.2}

As in the proof of Theorem 3.1, we first focus on the case where $a_{i j}=r_{i j}^{\alpha}$, for $\alpha \leq 1$ but we consider arbitrary minimum distance networks on the plane. We focus on bounding the first term in (6) and follow exactly the same steps up until (19). Here, we observe that (see [4, , Corollary 3.2]), since we are dealing with networks on a plane, there are at most $(2 \pi+12) l_{j k}$ nodes $j$ that share a common value of $l_{j k}$, for fixed $k$, and similarly there are at most $(2 \pi+12) l_{k i^{*}}$ nodes $k$ that share a common value of $l_{k i^{*}}$, for fixed $i^{*}{ }^{5}$ Thus, (19), the bound on $B_{1}$, now becomes

$$
\sum_{l_{k i^{*}=1}}^{\infty} \frac{(2 \pi+12) l_{k i^{*}}}{\left(1+r_{m} l_{k i^{*}}\right)^{\delta-\frac{\alpha}{4}}}\left\{\sum_{l_{j k}=l_{k i^{*}}}^{\infty} \frac{2(2 \pi+12) l_{j k}}{\left(1+r_{m} l_{j k}\right)^{2 \delta-\frac{3}{2} \alpha}}\right\}^{\frac{1}{2}} .
$$

Following the same steps as in the proof of Theorem 3.1, we obtain

$$
B_{1}<\frac{\sqrt{2}(2 \pi+12)^{\frac{3}{2}}}{r_{m}^{2 \delta-\alpha}}\left\{\zeta\left(2 \delta-\alpha-\frac{3}{2}\right)+K_{2}(\alpha, \delta)^{\frac{1}{2}} \zeta(2 \delta-\alpha-2)\right\}
$$

where $K_{2}(\alpha, \delta):=\left(2 \delta-\frac{3}{2} \alpha-2\right)^{-1}$. The above bound on $B_{1}$ is valid for $\delta>\frac{3}{2}+\frac{\alpha}{2}$. We can make similar observations for bounding the term $B_{2}$ to obtain

$B_{2}<\frac{2^{\delta-\frac{\alpha}{4}+\frac{1}{2}}(2 \pi+12)^{\frac{3}{2}}}{r_{m}^{2 \delta-\alpha}}\left\{\zeta\left(\delta-\frac{\alpha}{4}-1\right)\right.$

${ }^{5}$ Similarly to the linear case, $i^{*}$ is here set to be the "center" node. We can always add more nodes to the configuration to make any node we choose the center node.

$$
\left.+K_{2}(\alpha, \delta)^{\frac{1}{2}} c_{2}(\alpha, \delta)\left[\zeta\left(\delta-\frac{\alpha}{4}-1\right)-\zeta(2 \delta-\alpha-2)\right]\right\}
$$

where

$$
c_{2}(\alpha, \delta) \stackrel{\text { def }}{=}\left(1-2^{\frac{3}{2} \alpha-2 \delta+2}\right)^{\frac{1}{2}}\left(1-2^{\frac{3}{4} \alpha-\delta+1}\right)^{-1}
$$

and the bound is valid for $\delta>2+\frac{\alpha}{4}$.

Combining the two bounds on $B_{1}$ and $B_{2}$, we establish the scaling of the first term in (6). The second term exactly corresponds to the no-CSI bound of (5), whose scaling law is directly obtained from [4], Corollary 3.2. Hence, we obtain the scaling law of $C_{f}$ when $f(r)=r^{\alpha}, \alpha \leq 1$ for planar minimum distance networks. To extend this to generalized transport capacity, we make the same observation as in the proof of Theorem 3.1. Hence, we establish Theorem 3.2.

\section{ACKNOWLEDGMENT}

The authors thank D. Shah for an interesting discussion; in particular, this led to the development in Section II-A2.

\section{REFERENCES}

[1] T. M. Cover and J. Thomas, Elements of Information Theory. New York: Wiley, 1991.

[2] M. M. Deza and M. Laurent, "Geometry of cuts and metrics," in Algorithms and Combinatorics. New York: Springer-Verlag, 1997, vol. 15.

[3] P. Gupta and P. R. Kumar, "Capacity of wireless networks," IEEE Trans. Inf. Theory, vol. 46, no. 2, pp. 388-401, Mar. 2000.

[4] A. Jovičić, P. Viswanath, and S. R. Kulkarni, "Upper bounds to transport capacity of wireless networks," IEEE Trans. Inf. Theory, vol. 50, no. 11, pp. 2555-2565, Nov. 2004.

[5] O. Lévêque and E. Telatar, "Information theoretic upper bounds on the capacity of large extended ad hoc wireless networks," IEEE Trans. Inf. Theory, vol. 51, pp. 858-865, Mar. 2005.

[6] R. T. Rockafellar, Convex Analysis. Princeton, NJ: Princeton Univ. Press, 1971.

[7] L.-L. Xie and P. R. Kumar, "A network information theory for wireless communication: Scaling laws and optimal operation," IEEE Trans. Inf. Theory, vol. 50, pp. 748-767, May 2004.

[8] F. Xue, L.-L. Xie, and P. R. Kumar, "The transport capacity of wireless networks over fading channels," IEEE Trans. Inf. Theory, vol. 51, pp. 834-847, Mar. 2005.

[9] A. Gupta, I. Newman, Y. Rabinovich, and A. Sinclair, "Cuts, trees and $l_{1}$-embeddings of graphs," Combinatorica, vol. 24, pp. 233-269, 2004. 